\title{
A NOTE ON CHRISTOFIDES' CONJECTURE REGARDING WANG'S PREMIUM PRINCIPLE ${ }^{1}$
}

\section{BY}

WANG JING-LONG

East China Normal University, Shanghai, China

\section{INTRODUCTION}

Young (1999) discussed the conjecture proposed by Christofides (1998) regarding the premium principle of Wang $(1995,1996)$. She shows that this conjecture is true for location-scale families and for certain other families, but false in general. In addition Young (1999) states that it remains an open problem to determine under what circumstances Wang's premium principle reduces to the standard deviation (SD) premium principle.

In this paper we will provide further discussion of this problem. We will show that, for a fixed distortion, the natural set on which Wang's premium principle can reduce to the SD premium principle is and only is the union of location-scale families which satisfies some condition. Furthermore, it will be shown that the natural set is and only is a location-scale family if Wang's premium principle can be reduced to the SD premium principle for any distortion.

\section{RESULTS}

As we all know, the standard deviation premium principle applied to a random variable $X$ gives the premium

$$
E(X)+\lambda \sqrt{\operatorname{Var}(X)}
$$

for some $\lambda>0$, and Wang's $(1995,1996)$ premium principle gives the premium

$$
H_{g}(x)=\int_{-\infty}^{0}\left\{g\left[S_{X}(t)\right]-1\right\} d t+\int_{0}^{\infty} g\left[S_{X}(t)\right] d t
$$

\footnotetext{
1 Project 19831020 Supported by National Natural Science Foundation of China. 
where $S_{X}(t)=P(X>t)$ is the decumulative distribution function (ddf) of $X$, and the distortion $g$ is a non-decreasing function from $[0,1]$ onto itself. Suppose that Wang's premium principle reduces to the SD premium principle for a set $F$ of distributions, i.e. for any $X \in F$, we have

$$
E(X)+\lambda \sqrt{\operatorname{Var}(X)}=H_{g}(x)
$$

Hence for any $X, Y \in F$, we have

$$
\frac{H_{g}(X)-E(X)}{\sqrt{\operatorname{Var}(X)}}=\frac{H_{g}(Y)-E(Y)}{\sqrt{\operatorname{Var}(Y)}}
$$

Further, such a set is called the natural set for the given $g$, if for any $X \in F$ and $Y \notin F$, the following condition is satisfied:

$$
\frac{H_{g}(X)-E(X)}{\sqrt{\operatorname{Var}(X)}} \neq \frac{H_{g}(Y)-E(Y)}{\sqrt{\operatorname{Var}(Y)}}
$$

As Young (1999) shows, Wang's premium principle reduces to the SD premium principle on the location-scale family $\Pi=\{X=\mu+\sigma \cdot Z: \mu \in \mathbf{R}, \sigma>0\}$, where $Z$ is a random variable. We call $Z$ the underlying distribution of this location-scale family $\Pi$. In fact, any a distribution of $\Pi$ can be regarded as the underlying distribution of $\Pi$. Obviously, if $F$ is a natural set, $\Pi \subseteq F$ for $Z \in F$. That means the location-scale family with the underlying distribution being a element of the natural set $F$ for which Christofides conjecture is true is a subset of $F$.

Christofides (1998) conjectures that for a parametric family of distributions with constant skewness Wang's premium principle reduces to the SD premium principle. Young (1999) shows that this conjecture is false in general. Otherwise, if Wang's premium principle reduces to the SD premium principle for a parametric family of distribution, is this family with constant skewness? It is also not true in general. See the following examples.

Following and example in Young (1999), let $X$ be a random variable have a two-sided exponential distribution with parameters $\alpha=1, \beta=2.27466$ and $w=0.1$. Its ddf and skewness are, respectively:

$$
\begin{aligned}
S_{X}(t) & = \begin{cases}w+(1-w)\left(1-e^{3 t}\right), & t<0 ; \\
w e^{-\alpha t}, & t \geq 0 .\end{cases} \\
\text { Skew } X & =\frac{E\left\{[X-E(X)]^{3}\right\}}{[\operatorname{Var}(X)]^{\frac{3}{2}}}=1.84166
\end{aligned}
$$

Let the distortion $g(p)=p^{0.5}$. Then

$$
\frac{H_{g}(X)-E(X)}{\sqrt{\operatorname{Var}(X)}}=1.02386
$$


Let $Y$ have a Pareto distribution with parameters $\alpha=43.41704$ and $\beta$. Its ddf, expectation, variance and skewness are the follows respectively:

$$
\begin{aligned}
S_{Y}(t) & =P(Y>t)=\left(\frac{\beta}{1+\beta}\right)^{\alpha} \\
E(Y) & =\frac{\beta}{\alpha-1}, \quad \operatorname{Var}(Y)=\frac{\alpha \beta^{2}}{(\alpha-1)^{2}(\alpha-2)} \\
\operatorname{Skew}(Y) & =\frac{2(\alpha+1) \sqrt{\alpha-2}}{\sqrt{\alpha}(\alpha-3)}=2.146777 \neq \operatorname{Skew}(X)
\end{aligned}
$$

Let the distortion be the same as above, then

$$
\frac{H_{g}(Y)-E(Y)}{\sqrt{\operatorname{Var}(Y)}}=\left(\frac{\alpha}{\alpha-2}\right)^{\frac{1}{2}}=1.02386=\frac{H_{g}(X)-E(X)}{\sqrt{\operatorname{Var}(X)}}
$$

Hence, for the distortion $g(p)=p^{0.5}$, Wang's premium principle can reduce to the SD premium principle for the union $\Pi_{1} \cup \Pi_{2}$, where

$$
\begin{aligned}
& \Pi_{1}=\{\mu+\sigma \cdot X: \mu \in \mathbf{R}, \sigma>0\} \\
& \Pi_{2}=\{\nu+\tau \cdot Y: \nu \in \mathbf{R}, \tau>0\}
\end{aligned}
$$

are the location-scale families with underlying distributions $X$ and $Y$ respectively. Thus, Wang's premium principle reduces to the SD premium principle for a parametric family of distribution $\Pi_{1} \cup \Pi_{2}$ whose members do not all have the same skewness.

From the preceeding discussion we get the following proposition.

\section{Proposition 1.}

For a fixed distortion $g$, the natural set on which Wang's premium principle reduces to the standard deviation premium principle is and only is the union of location-scale families:

$$
\cup_{i \in I} \Pi_{i}
$$

where $I$ is an index set, for any $i \in I, \Pi_{i}$ is a location-scale family: $\Pi_{i}=\left\{\mu_{i}+\sigma_{i} \cdot X_{i}: \mu_{i} \in \mathbf{R}, \sigma_{i}>0\right\}$, and their underlying distributions $X_{i}(i \in I)$ satisfy the following condition: for any $i, j \in I$ we have

$$
\frac{H_{g}\left(X_{i}\right)-E\left(X_{i}\right)}{\sqrt{\operatorname{Var}\left(X_{i}\right)}}=\frac{H_{g}\left(X_{j}\right)-E\left(X_{j}\right)}{\sqrt{\operatorname{Var}\left(X_{j}\right)}}
$$

Furthermore, the natural set is and only is a location-scale family if Wang's premium principle can reduce to the SD premium principle for any distortion. 


\section{Proposition 2.}

The natural set on which Wang's premium principle can reduce to the standard deviation premium principle for any distortion is and only is a location-scale family.

\section{Proof:}

Suppose that for any distortion Wang's premium principle can reduce to the SD premium principle on the set $F$ of distributions. Then for any $X, Y \in F$ and for any distortion $g$, we have

$$
\frac{H_{g}(X)-E(X)}{\sqrt{\operatorname{Var}(X)}}=\frac{H_{g}(Y)-E(Y)}{\sqrt{\operatorname{Var}(Y)}}
$$

Let

$$
U=\frac{X-E(X)}{\sqrt{\operatorname{Var}(X)}}, \quad V=\frac{Y-E(Y)}{\sqrt{\operatorname{Var}(Y)}}
$$

From equation (1), we have

$$
H_{g}(U)=H_{g}(V)
$$

because $H_{g}$ is location and scale equivariant. Denote the decumulative distribution functions of $U$ and $V$ by $S_{U}(t)$ and $S_{V}(t)$ respectively. Firstly, using proof by contradiction, we will show that $S_{U}(t)=S_{V}(t)$ when $t \geq 0$. Assume that there is a $t_{0} \geq 0$ so that $S_{U}\left(t_{0}\right)>S_{V}\left(t_{0}\right)$. Let $\alpha=S_{V}\left(t_{0}\right), 0 \leq \alpha<1$.

- Case 1.

Suppose that $\left\{t: S_{U}(t)=\alpha\right\} \neq \emptyset$

Let

$$
u_{1}=\inf \left\{t: S_{U}(t)=\alpha\right\}, \quad v_{1}=\inf \left\{t: S_{V}(t)=\alpha\right\}
$$

Because the non-increasing ddf is a right continuous function, $S_{U}\left(u_{1}\right)=S_{V}\left(v_{1}\right)=\alpha$, which implies that $v_{1} \leq t_{0}<u_{1}$. Let the distortion

$$
g(w)= \begin{cases}1, & \alpha<w \leq 1 \\ 0, & 0 \leq w \leq \alpha\end{cases}
$$

Then $H_{g}(U)=u_{1}$, and for $v_{1} \in(-\infty, \infty)$, it can be proved that $H_{g}(V)=v_{1}$. Hence $H_{g}(U) \neq H_{g}(V)$, contradicting equation (2).

- Case 2.

Suppose that $\left\{t: S_{U}(t)=\alpha\right\}=\emptyset$.

Then there is a $u_{0}$ so that $S_{U}\left(u_{0}-0\right) \geq \alpha>S_{U}\left(u_{0}\right)$ and $S_{U}(t)>\alpha$ when $t<u_{0}$. Obviously, $t_{0}<u_{0}$. Let the distortion be the same as above, then $H_{g}(U)=u_{0}, H_{g}(V)=v_{1}$, which implies $H_{g}(U) \neq H_{g}(V)$, again contradicting equation (2). 
Because a ddf is a non-increasing function, the set consisting of points at which the ddf is discontinuous (that is, $\{t$ : ddf isn't continuous at point $t\}$ ) is either a finite set or a countable set. Therefore, it is proved that $S_{U}(t)=S_{V}(t)$ when $t \geq 0$.

Now, we will show $S_{U}(t)=S_{V}(t)$ when $t<0$. Let $\tilde{U}=-U$ and $\tilde{V} \equiv-V$. Then the decumulative distribution functions of $\tilde{U}$ and $\tilde{V}$ are $S_{\tilde{U}}(t)=1-S_{U}(-t-0)$ and $S_{\tilde{V}}(t)=1-S_{V}(-t-0)$ respectively. From equation (2), we have $H_{g}(\tilde{U})=H_{g}(\tilde{V})$. According to the above result, $S_{\tilde{U}}(t)=S_{\tilde{V}}(t)$ when $t \geq 0$, which implies $S_{U}(t-0)=S_{V}(t-0)$ when $t \leq 0$. Hence $S_{U}(t)=S_{V}(t)$ when $t \leq 0$.

To sum up, $S_{U}(t) \equiv S_{V}(t)$. Hence, $U=V$ almost surely. The proposition is proved.

In fact, the assumption of this Proposition can be weakened: that is, the natural set on which Wang's premium principle can reduce to the standard deviation premium principle for any two-step-up distortion is and only is a location-scale family. Is and only is the natural set a location-scale family for any power distortion? This is a subject of future study.

\section{ACKNOWLEDGEMENTS}

I wish to thank the editors and referees for their valuable comments and corrections, which resulted in considerable improvement of my original paper.

\section{REFERENCES}

Christofides, S. (1998) Principle for risk in financial transactions. Proceedings of the GISG/ ASTIN Joint Meeting in Glasgow, Scotland. October, 1998, 2, 63-109.

WANG, S.S. (1995) Insurance pricing and increased limits ratemaking by proportional hazards transforms. Insurance: Mathematics and Economics 17, 43-54.

WANG, S.S. (1996) Premium calculation by transforming the layer premium density. ASTIN Bulletin 26, 71-92.

YounG, V.R. (1999) Discussion of Christofides' conjecture regarding Wang's premium principle. ASTIN Bulletin 29, 191-195.

WANG JING-LONG

Department of Statistics

East China Normal University

3663 Zhongshan Road (Northern)

Shanghai 200062

P.R. China 